\title{
The Equivalent T Circuit of the Induction Motor: Its Non-uniqueness and Use to the Magnetic Field Analyst
}

\author{
Kent R. Davey, Fellow, IEEE
}

\begin{abstract}
The equivalent $\mathbf{T}$ circuit of an induction motor is found in every engineering motors book and is becoming increasingly important with the popularity of vector control. The values used in the circuit are used to dictate the necessary gating signals for the pwm inverter. It is the de facto standard bridge between the field analyst and the motor controller. These parameters are not unique. Force, energy, voltage, and secondary power dissipation can be shown to relate to the product of synchronous reactance and the coupling coefficient of the rotor and stator. The mutual reactance, the secondary inductance, and secondary resistance are non-unique. Yet this surprising feature can be used as a solution integrity check for the field analyst.
\end{abstract}

Index Terms-coupling-coeficient, equivalent circuit, induction, motor

\section{BACKGROUND ON THE EQUIVALENT T CIRCUIT}

$\mathrm{I}^{\mathrm{n}}$ n the last 25 years, EEE has published 340 papers on the derivation of the equivalent induction motor circuit parameters. In the past 8 years, the Magnetics journal published 23 papers on this subject due in part to the popularity of vector motor control which depends on this circuit. In none of the papers does the non-uniqueness problem receive discussion, which is the first motivation of this paper. .

Cendes [1] outlined a helpful procedure for linking a finite element analysis to the circuit parameters; the stepped procedure makes the assumption that the mutual inductance dominates leakage, an unwarranted assumption for canned and linear induction motors. In a previous paper by this author [2], the uniqueness was removed by assuming an equivalence between the leakage on the rotor and the stator. In this paper, a prediction of the equivalent circuit parameters was derived from the voltage, slip, and torque at pull out. More recently, Nelatury [3] demonstrated a similar method using any three points on the torque speed curve; he too assumes the magnetizing inductance dominates leakage. Based on this assumption, Nelatury derives rotor resistance, the sum of the stator and rotor leakage reactance and the stator resistance. For canned rotors, Salon [4] assumes, after defense based on calculation, that the inductance of the secondary can be neglected. The reader will find that assumptions of this nature are always made before specifying equivalent circuit parameters. What is not mentioned is the non-uniqueness of the circuit; the equivalent circuit performance prediction will be just as accurate with multiple assumptions. Surprisingly, this information is not only useful to the field analyst in terms of evaluating solution integrity, but also in machine

Manuscript received January 15, 2006. This work was supported by the Office of Naval Research. K. R. Davey is with the Center for Electromechanics, 10100 Burnet Rd., Austin, Texas, 78758, USA (phone 512-232-1603; fax: 512-475-7700; e-mail: k.davey@mail.utexas.edu). optimization.

A second motivation for this paper is spurred from the popularity of space vector control. Codes for providing gate signals for three phase induction motors using pulse width modulation are now common place in industry [5]. The parameters requested in these standard control signals are the equivalent $\mathrm{T}$ circuit parameters. How do we, as field theoreticians, derive the best parameters for the motor controller? The surprising answer is that there are innumerable parameter values that can be supplied to the space vector algorithm, all of which will give identical performance.

The outline of this paper is as follows. First, the global quantities of interest, like torque, power dissipation, energy, and voltage will be derived in terms of the circuit parameters. They are represented in terms that are most convenient for the field analyst, i.e., current excitation. Second, the dependence of these quantities on slip is examined with the intent of showing the error of thinking that circuit parameters can be computed by changing speed. Contrary to popular thinking, the locked rotor and no load motor tests do not reveal unique circuit parameters, but only approximations under certain conditions. Thirdly, a procedure for choosing non-unique, but serviceable motor parameters is disclosed. Finally, the usefulness of these relations for solution integrity and design are discussed.

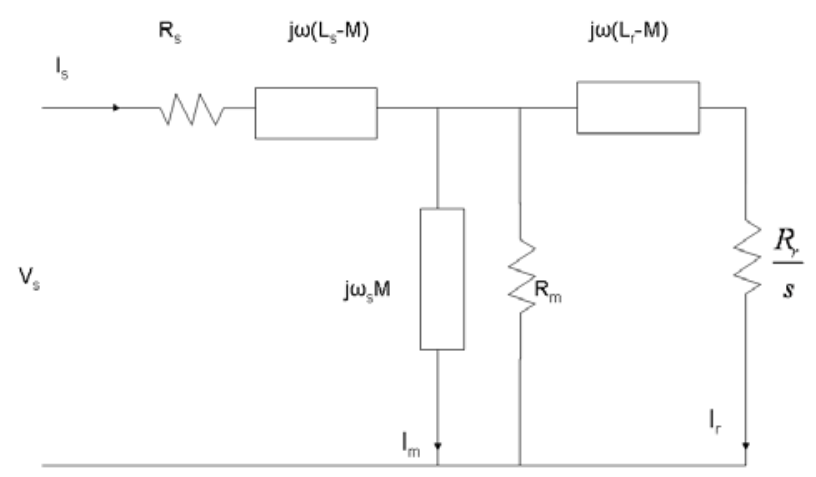

Fig. 1. One phase of the equivalent $\mathrm{T}$ circuit for an induction motor.

\section{THE CIRCUIT AND ITS RELATION TO GLOBAL PARAMETERS}

Among the more comprehensive formulations which show how to derive the equivalent induction motor circuit from fundamental field relations is that developed by Melcher [6]. Fig. 1 shows a single phase (voltage to neutral) of an induction motor. 
The representation is exact to the extent that magnetizing core loss and saturation remain unchanged through the operating range of the motor. The various inductances also show frequency dependence with mixed deep bar and shallow bar rotors. These subtleties simply cause the various parameters to have both current and frequency dependence. These effects and the required parameter refinement will not be considered in this paper.

The magnetizing inductance $M$ is typically defined in terms of the rotor and stator inductance through the coupling coefficient $k$, where $M=k \sqrt{L_{s} L_{r}}$.

For the moment it is convenient to consider the magnetizing resistance $R_{m}$ to be infinite; it is a parameter that is derived after the fact and is typically based on manufacturer's data characterized by the lamination geometry and material composition. The standard definition for slip $s$ is employed involving the difference of electrical speed $\omega_{s}$ and mechanical speed $\omega_{r}$, i.e., $s=\left(\omega_{s}-\omega_{r}\right) / \omega_{s}$. The torque for a $q$ phase induction motor is [7]

$$
T=q \frac{\left(\omega_{s}-\omega_{r}\right) M^{2} R_{r} I_{s}{ }^{2}}{R_{r}{ }^{2}+\left(\omega_{s}-\omega_{m}\right)^{2} L_{r}{ }^{2}}=q \frac{\omega_{s} M^{2} I_{s}{ }^{2} \frac{R_{r}}{s}}{\left(\frac{R_{r}}{s}\right)^{2}+\left(\omega_{s} L_{r}\right)^{2}} .
$$

At peak (pull out) torque, $\omega_{s} L_{r}=R / s$. It is useful to rewrite the peak torque in terms of this peak slip $s_{p}$ and the coupling coefficient $k$

$$
T=q \frac{s_{p}}{s} \frac{I_{s}^{2} k^{2} L_{s}}{\left(\left(\frac{s_{p}}{s}\right)^{2}+1\right)} .
$$

The peak torque follows as

$$
T_{p}=\frac{q}{2} k^{2} L_{s} I_{s}^{2} \text {. }
$$
is

$$
V=j \omega_{s} L_{s} I_{s}+\frac{\omega_{s}{ }^{2} M^{2} I_{s} \frac{R_{r}}{s}}{\left(\frac{R_{r}}{s}\right)^{2}+\omega_{s}{ }^{2} L_{r}{ }^{2}}-j \frac{\omega_{s}^{3} M^{2} L_{r} I_{s}}{\left(\frac{R_{r}}{s}\right)^{2}+\omega_{s}{ }^{2} L_{r}{ }^{2}} .
$$

This voltage can be rewritten in terms of peak slip as

$$
V=j \omega_{s} L_{s} I_{s}+\left(\frac{\omega_{s} L_{s} k^{2} I_{s} \frac{s}{s_{p}}}{1+\left(\frac{s}{s_{p}}\right)^{2}}\right)\left(1-j\left(\frac{s}{s_{p}}\right)\right) .
$$

The power dissipated in the rotor is easily calculated in a numerical code by integrating $\sigma \vec{E} \cdot \vec{E}$ over the volume of the rotor. This quantity follows from the circuit by squaring the portion of the current that passes through the rotor and multiplying it by the rotor resistance.

$$
P=q \frac{I_{s}{ }^{2} \omega_{s}{ }^{2} M^{2}}{\left(\omega_{s} L_{r}\right)^{2}+\left(\frac{R_{r}}{s}\right)^{2}} R_{r}=q \frac{\omega_{s} k^{2} L_{s} I_{s}{ }^{2} \frac{s^{2}}{s_{p}}}{\left(1+\left(\frac{s}{s_{p}}\right)^{2}\right)} .
$$

Energy is the last global quantity of interest. The field theoretician computes this as

$$
W=\int_{V} \vec{A} \cdot \vec{J} d V .
$$

Designate $I_{r}$ and $I_{m}$ as the current through the magnetizing inductance and the rotor resistance path.

$$
\begin{aligned}
& I_{r}=I_{s} \frac{j \omega_{s} M}{j \omega_{s} L_{r}+\frac{R_{r}}{s}}, \\
& I_{m}=I_{s} \frac{j \omega_{s}\left(L_{r}-M\right)+\frac{R_{r}}{s}}{j \omega_{s} L_{r}+\frac{R_{r}}{s}} .
\end{aligned}
$$

The energy stored in the machine at any instant is

$$
W=\frac{1}{2}\left(L_{s}-M\right) I_{s}{ }^{2}+\frac{1}{2} M I_{m}{ }^{2}+\frac{1}{2}\left(L_{r}-M\right) I_{r}{ }^{2} .
$$

Inserting (8) and (9) into (10) and substituting the coupling coefficient for $M$, and $s_{p} \omega_{s} L_{r}$ for $R_{r}$ gives the result

$$
W=\frac{1}{2} I_{s}^{2} L_{s} \frac{\left(1-k^{2}+\left(\frac{s_{p}}{s}\right)^{2}\right)}{1+\left(\frac{s_{p}}{s}\right)^{2}} .
$$

\section{THE PREFERED METHOD OF INFERRING EQUIVALENT CIRCUIT PARAMETERS}

Except for a small class of axial and transverse flux machines, induction motor designs are analyzed in two dimensions. In parametric analysis, torque, voltage, and power dissipation are readily computed in any commercial finite element program as a function of slip. The resistance of the stator winding is dependent on the slot packing fraction and end turn length; this quantity can be computed analytically. Of the remaining quantities, only $L_{s}$ follows straightaway from field analysis. It is computed by one of two means. Perhaps the simplest is to set the slip to zero and compute the flux $\Phi$ linking each phase of the stator winding.

$$
L_{s}=\frac{\Phi(s=0)}{I_{s}} \text {. }
$$

If a parametric scan on slip is performed with fixed excitation current, the stator inductance can also be computed using the real and imaginary components of the phase voltage at peak torque as

$$
L_{s}=\frac{\mathfrak{I}\left(V_{s}\left(s=s_{p}\right)\right)+\mathfrak{R}\left(V_{s}\left(s=s_{p}\right)\right)}{\omega_{s} I_{s}} .
$$

A careful examination of (2), (5), (6), and (11) should convince the reader that this is as far as one can go, i.e., no 
other circuit parameters can be determined without assumptions. Both the coupling coefficient $k$ and the peak slip value $s_{p}$ are easily computed, but not $M, L_{r}$, or $R_{r}$. For example $k$ can be computed using the peak torque value from (3) as

$$
k=\sqrt{\frac{2 T_{p}}{q I_{s}^{2} L_{s}}} .
$$

Analyzing the machine at different rotation values does not solve this problem. The field analyst can artificially change the conductivity of the rotor bars thinking the change will reveal direct information about $R_{r}$. This, however, only changes the location of $s_{p}$.

\section{A. The Root of the Non-uniqueness}

The reader will observe that the global quantities computed analytically return information about the ratio of $M$ and $L_{r}$ as well as the ratio of $L_{r}$ and $R_{r}$, but not the quantities themselves. At the root of the problem is the physics involved if the rotor is wound with multiple turns. Two rotors with the same amount of copper but with different turns, i.e., one made as a wound rotor, will perform identically. Both motors will deliver the same torque, induce the same voltage at slip and excitation, exhibit the same energy storage, and dissipate the same loss on the rotor. The field analyst can supply the motor controller two different circuits with a three parameter change and exactly predict torque, voltage, peak slip, power dissipation, and energy.

\section{B. Practical Solution for the Motor Controller}

For some time this author has fretted with the dilemma of how to compute the "best parameter fit" that is exactly right for a machine. One non-recommended approach is to solve for the fields, freeze the permeability everywhere, excite only the rotor with the same currents, compute the system energy, and finally divide this energy by $1 / 2 I_{r}^{2}$. Even here it is not exactly clear what value to assign for $I_{r}$. The rotor current $I_{r}$ is dispersed over multiple rotor bars. This is a useless exercise. It is a useless exercise because no global quantity is affected by an arbitrary assignment of one of the quantities $L_{r}, R_{r}$, or $M$.

A number of solutions exist, the most practical of which is to arbitrary assign $L_{r}=L_{s}$. A consequence of this assignment is that the leakage inductance for the rotor and stator become identical. The remaining quantities follow from $k$ and $s_{p}$ as

$$
\begin{aligned}
& M=k L_{s} \\
& R_{r}=s_{p} \omega_{s} L_{s}
\end{aligned} .
$$

\section{The Usefulness of this Information to the Field Analyst}

It should be clear that the quantity $k^{2} L_{s}$ is unique because it can be easily linked to torque, voltage, power dissipation, and energy. Designating peak slip as $s_{p}$, it can be shown that

$$
k^{2} L_{s}=\frac{T}{q I_{s}^{2}} \frac{s_{p}}{s}\left(1+\left(\frac{s}{s_{p}}\right)^{2}\right),
$$

$$
\begin{aligned}
& k^{2} L_{s}=\frac{P}{q I_{s}^{2}} \frac{s_{p}}{s^{2}}\left(1+\left(\frac{s}{s_{p}}\right)^{2}\right), \\
& k^{2} L_{s}=\frac{\Re\left(V_{s}\right)}{I_{s}} \frac{s_{p}}{s}\left(1+\left(\frac{s}{s_{p}}\right)^{2}\right) .
\end{aligned}
$$

A $4^{\text {th }}$ equation can be written for total energy using (11). These become excellent solution integrity checks, but note that new information is not obtained by changing slip.

For linear motors, thrust force $F$ replaces torque as the chief global quantity of interest; it involves the motor's wavelength $\lambda$. Equations (1) and (16) become replaced with

$$
\begin{aligned}
& F=\frac{2 \pi q}{\lambda} \frac{\omega_{s} M^{2} I_{s}{ }^{2} \frac{R_{r}}{s}}{\left(\frac{R_{r}}{s}\right)^{2}+\left(\omega_{s} L_{r}\right)^{2}}=\frac{2 \pi q}{\lambda} \frac{s_{p}}{s} \frac{I_{s}{ }^{2} k^{2} L_{s}}{\left(\left(\frac{s_{p}}{s}\right)^{2}+1\right)}, \\
& k^{2} L_{s}=\frac{F \lambda}{2 \pi q I_{s}^{2}} \frac{s_{p}}{s}\left(1+\left(\frac{s}{s_{p}}\right)^{2}\right) .
\end{aligned}
$$

\section{PRACTICAL ADVISE FOR USING THESE PRINCIPLES}

There are three recommendations that flow from this work. First, use easily computed global quantities computed from boundary element and finite element codes to predict equivalent circuit parameters.

\section{Linear Induction Motor Stator}

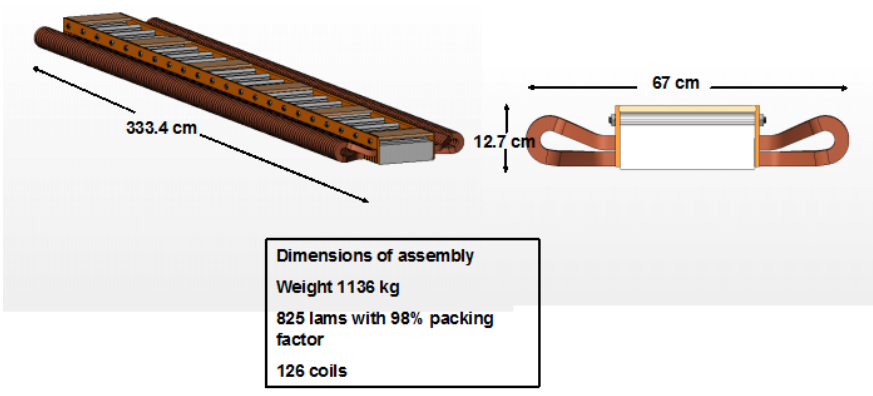

Fig. 2. Linear induction motor designed and modeled using these methods.

Second, use the redundant expressions for $k$ or $k^{2} L_{s}$ to serve as a check on the integrity of your solution. Be aware that real machines will exhibit small variations with slip due to deep rotor bar effects and saturation. Consider the $133 \mathrm{~cm}$ long linear induction motor shown in Fig. 2. The rotor is a $6.35 \mathrm{~cm}$ sheet of 3003 aluminum atop a $19 \mathrm{~cm}$ sheet of 1010 steel. Entry and exit effects complicate the analysis of global quantities. Fig. 3 shows a comparison of the thrust computed from boundary element codes with that computed from the procedure recommended above. The trace annotated "circuit 
using thrust" uses (20) to compute $k$, while "circuit using voltage” uses (18).

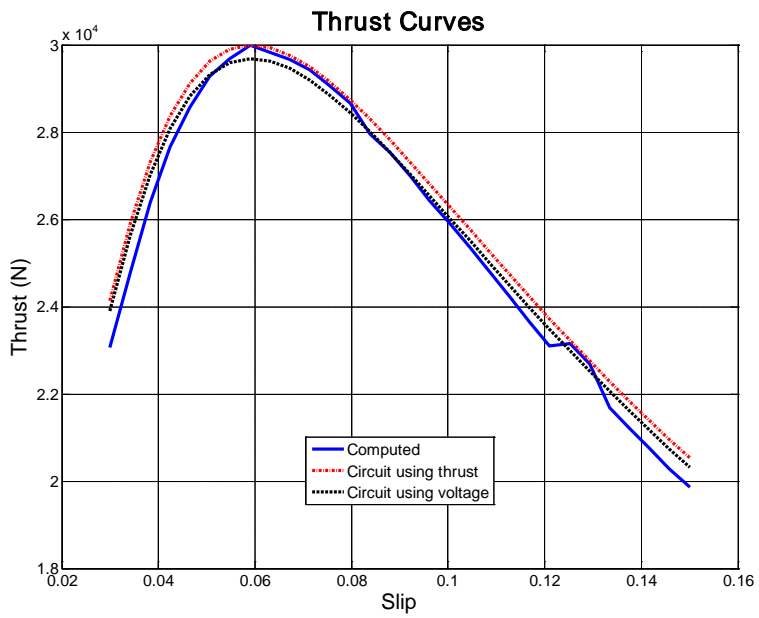

Fig. 3. Comparison of thrust curves computed from a boundary element code and from equivalent circuit parameters.

Table I shows the variation in coupling coefficient for this motor as computed using various methods. The reader should expect the largest variation when the slip is changed due to skin depth variation in the rotor bars.

Table I Variation of coupling coefficient computed using different parameters

\begin{tabular}{|l|c|}
\hline Method & Coupling Coefficient k \\
\hline Peak Torque & 0.8468 \\
\hline Voltage & 0.8425 \\
\hline Rotor Power dissipation & 0.8459 \\
\hline Torque at minimum slip & 0.8276 \\
\hline Torque at maximum slip & 0.8329 \\
\hline
\end{tabular}

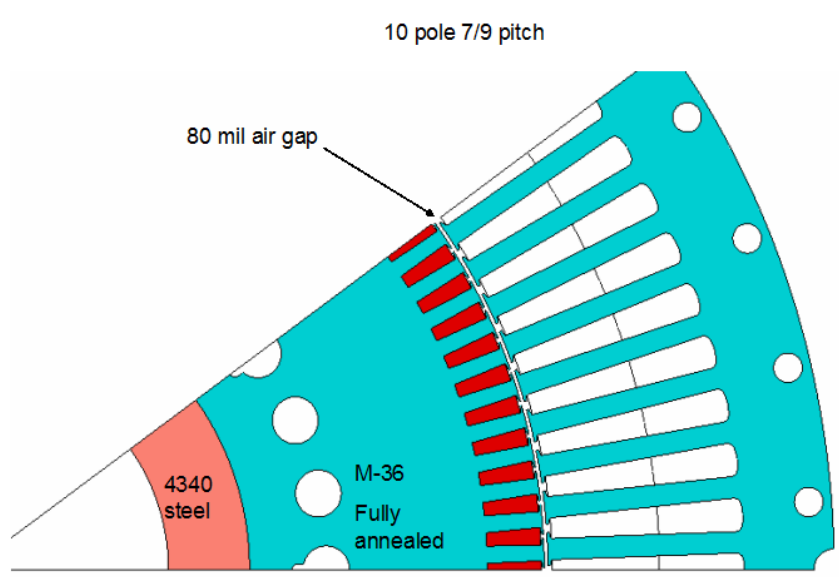

Fig. 4. Ten pole top drive with a $101.6 \mathrm{~cm}$ diameter.

Third, be aware that high torque density or thrust density machines are more problematic; the equivalent circuit parameter performance will deviate from the field theory computed values with slip and excitation change.

Fig. 4 shows an induction motor design for a very high torque density application, with tight diameter constraints. Fig. 5 shows the comparison of actual and equivalent circuit predicted torque. These parameters were computed at peak. Slip dependent inductance values would solve this problem, but the controller models are typically not equipped to handle this refinement. The pragmatic solution is to simply choose a slip off of peak in the parameter determination, i.e., to adopt a compromise fit. In very high density machines, it might be necessary to select $k$ from off peak slip quantities to maintain reasonable values for $k<1$.

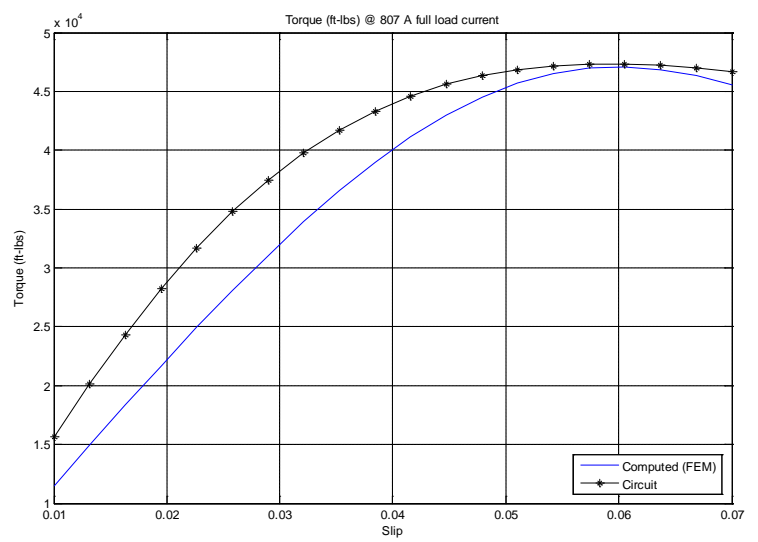

Fig. 5. Torque - speed curve for Motor A at full rated current.

\section{CONCLUSIONS}

With the advent of space vector control of industrial induction motors it is necessary for the field analyst and motor designer to supply equivalent circuit models with their designs. These parameters are not unique. One of the parameters $R_{r}, L_{r}$, or $\mathrm{M}$ is a discretionary quantity; all global quantities of concern to the controller will be unaffected. Global quantities delivered from finite element or boundary element codes should be used to derive these equivalent circuit parameters using the most convenient assignment. One of the most convenient choices is to set $\mathrm{L}_{\mathrm{r}}=\mathrm{L}_{\mathrm{s}}$. The redundancies on the coupling coefficient $\mathrm{k}$ serve as a useful index for quantifying the integrity of the field solution.

\section{REFERENCES}

[1] Z. Ping, J. Gilmore, Z. Badics, and Z. J. Cendes, "Finite element analysis of induction motors based on computing detailed equivalent circuit parameters," IEEE Trans. Mag., vol. 34, no. 5, pp. 3499-3502, Sept. 1988.

[2] K. R. Davey, "Predicting induction motor circuit parameters," IEEE Trans. on Mag., vol. 38, no. 4, pp. 1774-1779, July 2002.

[3] S. R. Nelatury, "Uniqueness of torque speed characteristics of an induction motor," IEEE Trans. on Mag., vol. 40, no. 5, pp. 3431-3433, Sept. 2004.

[4] L. T. Ergene and S. J. Salon, "Determining the equivalent circuit parameters of canned solid-rotor induction motors," IEEE Trans. on Mag., vol. 41, no. 7, pp. 2281-2286, July 2005.

[5] B. K. Bose, Modern Power Electronics and AC Drives, $1^{\text {st }}$ ed., New Jersey: Prentice-Hall, 2001.

[6] J. Melcher, Continuum Electromechanics, Cambridge: MIT Press, 1981, pp. 6.6-6.9.

[7] H. H. Woodson and J. R. Melcher, Electromechanical Dynamics, vol. 1, New York: Wiley, 1968, pp. 131-135. 